\title{
An All Optical Addressing Circuit: Experimental Results and Scalability Analysis
}

\author{
Donald M. Chiarulli, Member, IEEE, Robert M. Ditmore, Steven P. Levitan, Member, IEEE, and
}

Rami G. Melhem, Member, IEEE

\begin{abstract}
In this paper, we present results from a demonstration of both single and parallel selection in a one of four optical addressing circuit operating at $250 \mathrm{MHz}$ using coincident pulse addressing. We then present an analysis of power distribution in two different tapped fiber structures. Based on our results, we discuss issues of scalability with respect to synchronization and power distribution in larger systems.
\end{abstract}

\section{INTRODUCTION}

$\mathrm{T}$ WO properties of optical signals, unidirectional propagation and predictable path delay, make it possible to devise logic systems in which information is encoded as the relative timing of two optical signals. Coincident pulse addressing is an example of such a system. In this technique, the address of a detector site is encoded as the delay between two optical pulses which traverse independent optical paths to the detector. The delay is encoded to correspond exactly to the difference between the two optical path lengths. Thus, pulse coincidence, a single pulse with power equal to the sum of the two addressing pulses, is seen at the selected detector site. Other detectors along the two optical paths for which the delay did not equal the difference in path length, detect both pulses independently, separated in time.

Stated more formally, consider an optical fiber of length $L$ with two optical pulse sources, $P_{1}$ and $P_{2}$ coupled to each end. Each source generates pulses of width $\tau$ and height $h$. Define $l=\tau c_{g}$ where $c_{g}$ is the speed of light in the fiber. In other words $l$ is the length of fiber corresponding to the pulse width. Using $2 \times 2$ passive couplers, $n$ detectors, labeled $D_{1}$ through $D_{n}$, are placed in the fiber with the two tap fibers from each coupler cut to equal lengths and joined at the detector site. The location of each coupler/detector is carefully measured so that the $i$ th detector is located at $(L-(n+1) l) / 2+i l$, from the left end of the bus. The optical bus in the center of Fig. 1 shows such an arrangement for $n=3$. To uniquely address any detector, a specific delay between the pulses

Manuscript received March 25, 1991, revised July 23, 1991. This work was supported, in part, by the Air Force Office of Scientific Research under Grant number: AFOSR-89-0469.

D. M. Chiarulli and R. G. Melhem are with the Department of Computer Science, University of Pittsburgh, Pittsburgh, PA 15260

R. M. Ditmore is with Intergraph Corporation, Huntsville, AL.

S. P. Levitan is with the Department of Electrical Engineering, University of Pittsburgh, Pittsburgh, PA 15261.

IEEE Log Number 9103633. generated by $P_{1}$ and $P_{2}$ is chosen. If this delay is $(n-2 i$ $+1) \tau$, the two pulses will be coincident at detector $D_{i}$.

The same technique can be generalized to support parallel selections. If the $P_{1}$ source generates a single pulse at time $t_{r}$ and the source $P_{2}$ generates a series of pulses at times $t_{i}, i \in\{1, \cdots, n\}$ with each $t_{i}$ timed relative to $t_{r}$, then, according to the addressing equation above, to select a specific detector $i$ each $t_{i}$ will be in the range $-(n$ $-1) \tau \leq t_{r}-t_{i} \leq(n-1) \tau$. Therefore, any or all of the $i$ detectors can be uniquely addressed by a positionally distinguishable pulse from source $P_{2}$. For convenience, this pulse train is referred to as the select pulse train and the single pulse emanating from $P_{1}$ is called the reference pulse. Since the length of the select pulse train is $n$, and each pulse in the return to zero encoding is separated by $2 \tau$, it follows that the system latency, $\sigma=2 n \tau$. Further, up to $n$ locations may be selected in parallel within a single latency period. Therefore, the system throughput is $\nu$ $=1 / 2 \tau$.

In previous papers, we have discussed the general application of coincident pulse techniques to both memory addressing and multiprocessor network applications [2], [3], [7], [8]. In this paper, we emphasize the practical limits on the applicability of this technique for large systems. In order to design large scale computer systems, we need to know the realistic limits on the speed, size, and cost of such systems. Our long term goal is to build highspeed multiprocessor interconnection networks using off the shelf optical components and tapped fiber busses.

Tapped fiber busses, those with one or more transmitter and multiple receivers, have been less widely adopted than simple point-to-point fibers, primarily because of scalability limits based on power distribution [9]. However, the recent development of low ratio passive couplers [5] and the prospect of fiber based optical amplifiers [4], [6] suggest a closer examination of the power distribution problem. Therefore, we have constructed a prototype system for conducting experiments from which we can extrapolate reasonable limits on the speed and size of practical multicomputer systems.

In this paper, we first present results from two laboratory experiments on a prototype coincident pulse addressing system. The two questions to be answered by the experiments are: how do synchronization error and power loss effect the scalability of such systems. Therefore, the first experimental is an examination of the coincident pulse 
power, as a function of the synchronization of the arriving pulses. The second experiment demonstrates our ability to select arbitrary subsets of the detectors with a select pulse train operating at $250 \mathrm{MHz}$. The ability to perform selections of multiple detectors is key to various computer system applications we have investigated. However, the second experiment highlights a more fundamental problem: the power loss due to the tapping couplers on the bus diminishes the ratio of coincident to noncoincident pulse heights for long bus structures. The two experiments contrast the temporal and physical scalability of coincident pulse systems and show that the dominant effects are, in fact, power distribution limits on the physical scale of these systems.

Section III expands on the power distribution issue with an analysis of power distribution in two tapped fiber network structures. The first is the same linear structure that we use in our experiments. The second is a dual-level structure that consists of a main fiber and a series of secondary distribution fibers from which power is tapped. We conclude with a discussion of the implications of these findings to the construction of large systems.

\section{EXPerimental Results}

Fig. 1 is a diagram of the prototype structure. The fiber bus consists of a length of multimode fiber tapped three times using Gould 10-dB fiber couplers. Select and reference bit patterns are generated by modulating the 4-ns pulse output of a Tektronix PG502 pulse generator, shown in the diagram as clock, with the output of two ECL shift registers, one for select, one for reference, at gates $\mathrm{G} 2$ and G3. Gates G1 and G4 simultaneously hold the diode current for laser diodes $\mathrm{P} 1$ and $\mathrm{P} 2$ respectively at threshold, while the outputs of G2 and G4 generate modulation current. The result is two, 4-bit, return to zero bit streams, which encode the information in each of the shift registers. As explained above, this allows us to select any subset of the three (and in later experiments four) detectors. The use of two shift registers allows us flexibility in the positioning of the reference pulse relative to the select pulse train.

\section{A. Pulse Synchronization}

In our first experiment, measurements were made to characterize the effect of synchronization error between the reference and select pulses on the power of the coincident pulse. Since this error can be characterized as a percentage of the pulse width, synchronization precision has a direct bearing on the absolute width and height of an addressing pulse that can be effectively detected.

A coincident pulse structure with three detectors was used, as shown in Fig. 1. This allowed detector $D_{2}$ to be located in the center of the bus resulting in exactly equal noncoincident pulse heights, as shown in the oscilloscope trace of Fig. 2.

The reference and select pulse trains were configured to select $D_{2}$. In each step of the experiment, synchroniz-

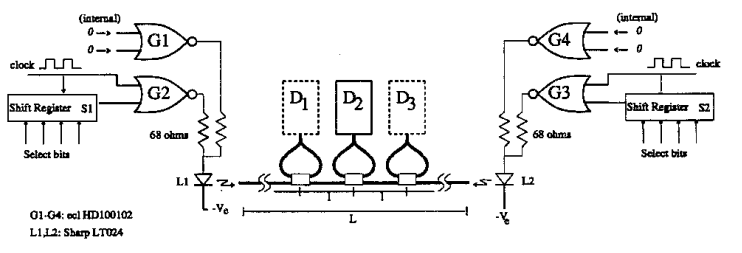

Fig. 1. Synchronization experiment.

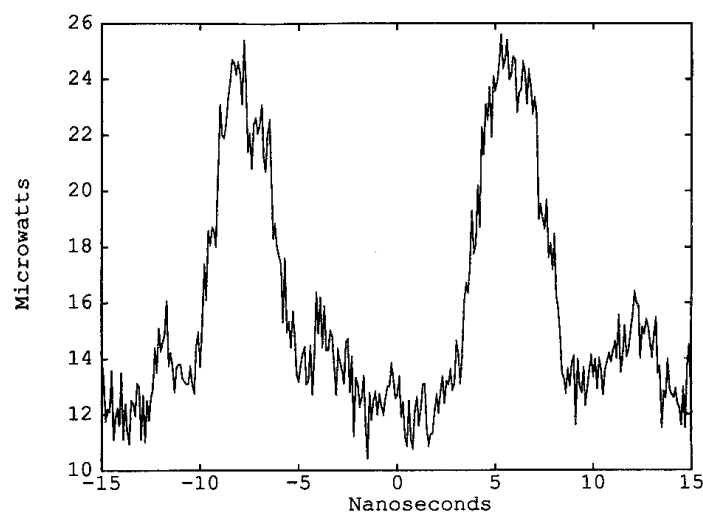

Fig. 2. Synchronization experiment waveform.

tion error was introduced by adding successively longer lengths of fiber to the ends of the bus. Length was added first on the reference pulse end of the bus, and then on the select pulse end of the bus.

Fig. 3 shows the reduction factor, $f$, of the coincident pulse power as a function of percent synchronization error. Percent synchronization error is the error, in time units, introduced by each length of fiber divided by the pulse width. In other words, pulses at perfect coincidence (synchronization error $=0$ ) yield a reduction factor of $f$ $=1.0$, which implies a coincident power equal to twice the single pulse power.

Synchronization error in either the select pulse, shown as positive error, or the reference pulse, shown as negative error, reduces this power by the factors shown. The solid line in Fig. 3 is the experimental result. The dotted line is an analytical result generated from the coincidence of two sinusoidal pulse waveforms. In both cases, the power falls off in roughly the shape of the coincident waveforms themselves.

In order to analyze this result, we must consider the sources of synchronization error. Assuming that manufacturing tolerances for electronic components and errors in fiber length measurements can be compensated for by tuning the system, the primary sources of synchronization error will be thermal variations in both the optical characteristics of the fiber and in the performance of electronic components as well as any jitter introduced by the electrical clock generators. For the former, recent results [10] have shown that the variability of the index of refraction of the fiber versus temperature is on the order of 40 $\mathrm{ps} / \mathrm{km}$-degree $\mathrm{C}$, and that this is the dominant tempera- 


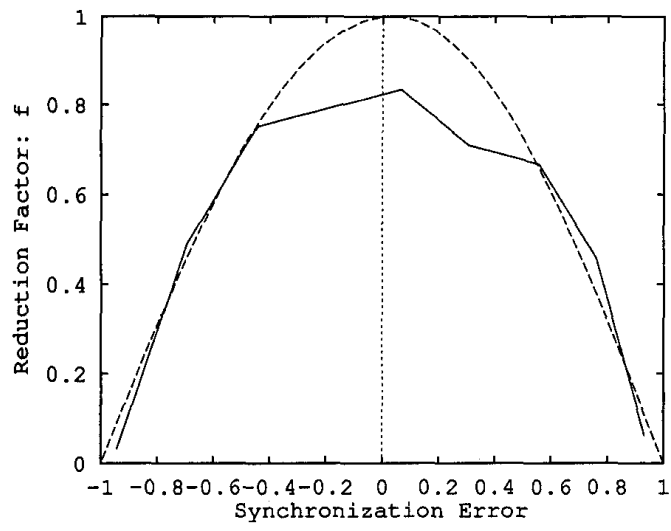

Fig. 3. Synchronization error reduction factor.

ture effect. This represents a very minor variation in effective optical path length. Obviously, jitter and thermal effects in the electronics will be the predominant sources of synchronization error.

However, from Fig. 3 we can see that a timing synchronization error of up to $50 \%$ only decreases the coincident pulse power to about $70 \%$ of its ideal value. Therefore, large variations (on the order of one half of a pulse width) in electronic pulse generation can be tolerated without significant degradation of the coincident signal. This result characterizes a temporal limit on scalability, based on a limit of achievable pulse widths. Timing errors of several hundred picoseconds are tolerable in gigahertz systems. Therefore, using off the shelf components operating in the one gigahertz range, $\tau=1 \mathrm{~ns}$ and a system throughput of $\nu=1 / 2 \tau=500 \times 10^{6}$ addressing operations per second is feasible.

The other primary limit, which we need to address, is optical power distribution. Since we are using a passive bus structure, the optical signals are not amplified at any point on the bus. Therefore, sufficient optical power must be available at each detector to individually discriminate coincidence from noncoincidence in the presence of selection pulses for other detectors and noise. This is the subject of the second experiment.

\section{B. Coincident Pulse Power}

Our second set of experiments were used to characterize the effect of detector position on the available coincident pulse power. A similar experimental setup was used, this time with four detectors, as shown in Fig. 4.

Figs. 5-8 show the output waveforms for detectors D1 and D3 for various selection patterns. Note that for each selection pattern (pair of waveforms) the experimental equipment was adjusted so that the absolute values of pulse heights for different selection patterns varied. Figs. 5 and 6 show coincident and noncoincident waveforms at detectors D1 and D3, respectively. Note that in both cases, the noncoincident waveforms (shown in (b)) are of unequal power. This is due to the fact that each pulse has passed through a different number of couplers and, hence,

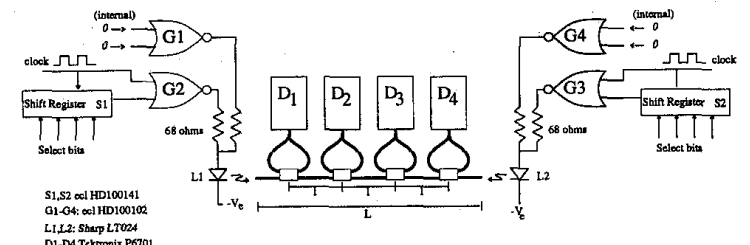

Fig. 4. Detector power experiment.

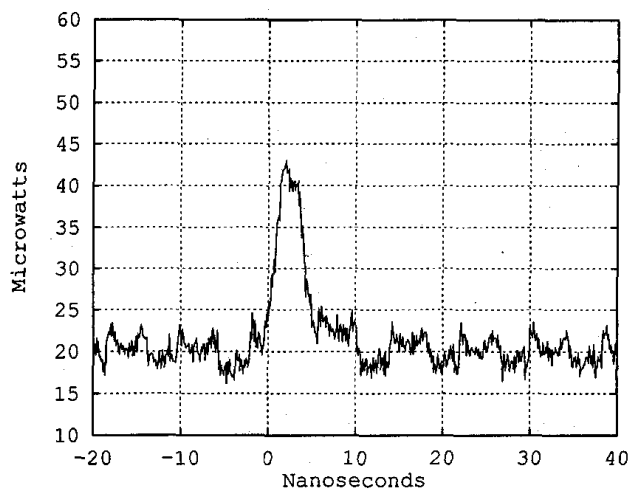

(a)

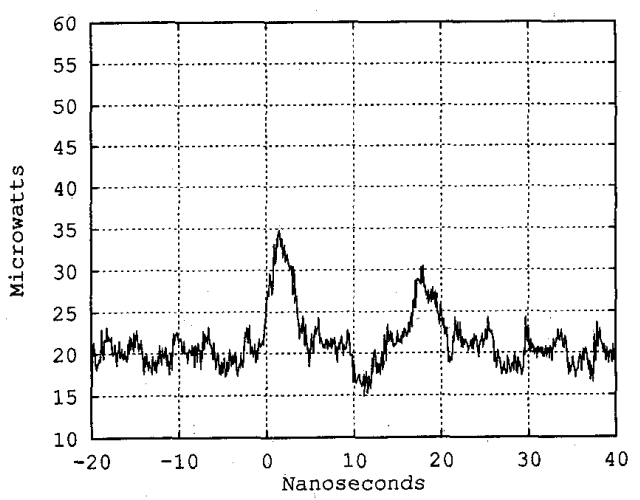

(b)

Fig. 5. (a) Selection of D1 measured at D1, (b) selection of D3 measured at D1

has become attenuated to different levels. This clearly shows that the relative power between coincident and noncoincident pulses is a function of the detector location.

Figs. 7 and 8 are examples of parallel selections. The waveform in Fig. 7(a) shows a parallel selection waveform at detector site $D_{3}$ for the selection of three detectors, including $D_{3}$. This incident waveform peak is comparable to the noncoincident waveform, in Fig. 7(b), in which $D_{3}$ has been removed from the set of selected locations. Similarly Fig. 8 shows parallel selection of all four detectors at sites $D_{1}$ and $D_{3}$.

To quantify the power degradation that we observed in these experiments, we define the amount of additional power in a coincident pulse relative to the largest noncoincident pulse seen by a detector as the power margin, $P m$. This is given as a fraction of the maximum noncoincident pulse power: 


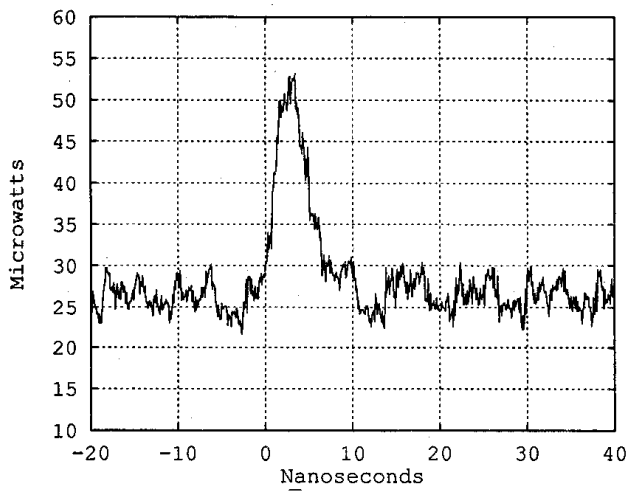

(a)

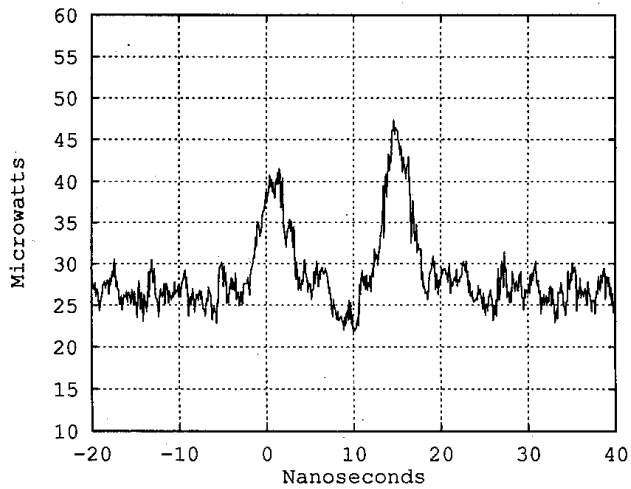

(b)

Fig. 6. (a) Selection of D3 measured at D3, (b) selection of D1 measured at D3.

$$
\begin{aligned}
P m & =\left(p_{1}+p_{2}-\max \left(p_{1}, p_{2}\right)\right) / \max \left(p_{1}, p_{2}\right) \\
& =\min \left(p_{1}, p_{2}\right) / \max \left(p_{1}, p_{2}\right) .
\end{aligned}
$$

$P m$ indicates the threshold level needed for a detector to discriminate between coincident and noncoincident pulses. That is, for each detector on the bus the threshold should be set to be at:

$$
((P m+1) \times \max (p 1, p 2)) / 2 .
$$

$P m$ has its maximum value, $P m=1$, at the center of the bus, where each pulse is at equal power, and coincidence is reflected as a doubling of power seen by the detector. It is at its minimum value at the ends of the bus. For all the selection experiments shown in Figs. 5 through 8, the power margin is in excess of $30 \%$. That is, coincident power is greater than $130 \%$ of peak single pulse power. This is measured at $D_{1}$, which is the leftmost detector on the bus.

In the next section we discuss the implications of power margin on scalability issues.

\section{Analytical Study of Power Distribution}

In this section, we present an analysis of power distribution in each of two tapped fiber network structures. The first is a simple linear structure with a single backbone

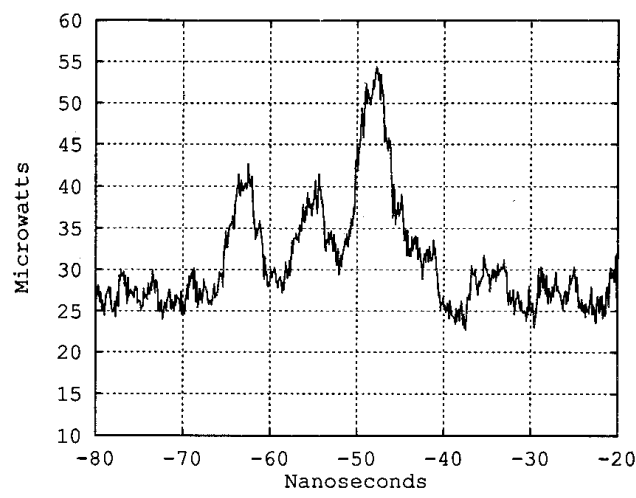

(a)

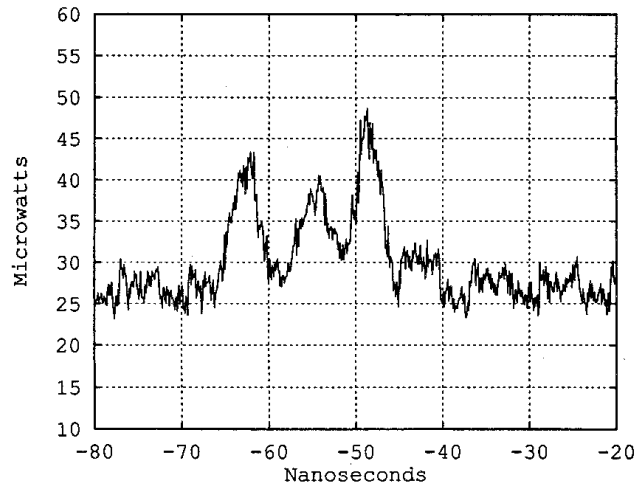

(b)

Fig. 7. (a) Selection of D1, D2, D3 measured at D3, (b) selection of D1, D2 measured at D3.

and a series of passive coupler taps, as used in the experiment above. The second is a dual level structure, which consists of a backbone fiber and a series of secondary distribution fibers from which power is tapped.

In this analysis, we use passive, bidirectional, $2 \times 2$, symmetric fiber couplers as shown in Fig. 9 [1], [5]. These are identical to the couplers we used in our previously discussed experiments, except that in our analysis we assume no excess loss in the couplers. Since the couplers are bidirectional, we arbitrarily let $A, B$ be the input ports and $A^{\prime}, B^{\prime}$ be the output ports. Equation (2) shows power distribution from the input to the output:

$$
\left(\begin{array}{l}
A^{\prime} \\
B^{\prime}
\end{array}\right)=\left(\begin{array}{ll}
r & (1-r) \\
(1-r) & r
\end{array}\right)\left(\begin{array}{l}
A B
\end{array}\right)
$$

where $r$ is the coupling ratio. Using these couplers, we now discuss the linear and dual level structures.

\section{A. The Linear Structure}

As is shown in Fig. 10, a linear bus consists of $n$ detectors (and $n$ couplers). Assuming two, unit height, pulses starting at opposite ends of the bus, and one type of coupler with a ratio of $r$, the optical power from each pulse $p_{i}^{1}$ and $p_{i}^{2}$ at detector $D_{i}$ is given by the equations:

$$
p_{i}^{1}=r^{(i-1)}(1-r), \quad p_{i}^{2}=r^{(n-i)}(1-r) .
$$




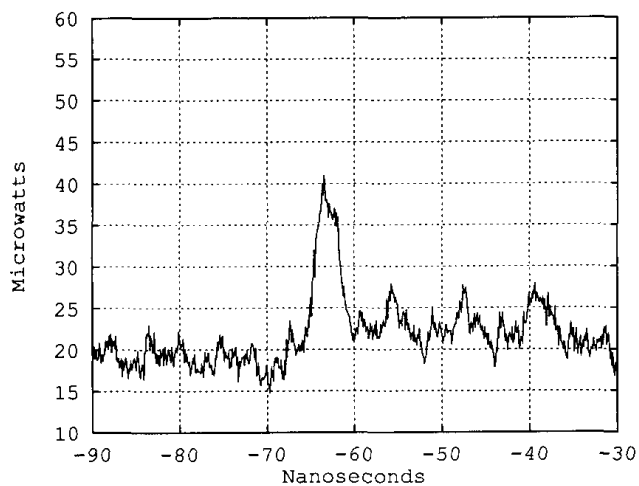

(a)

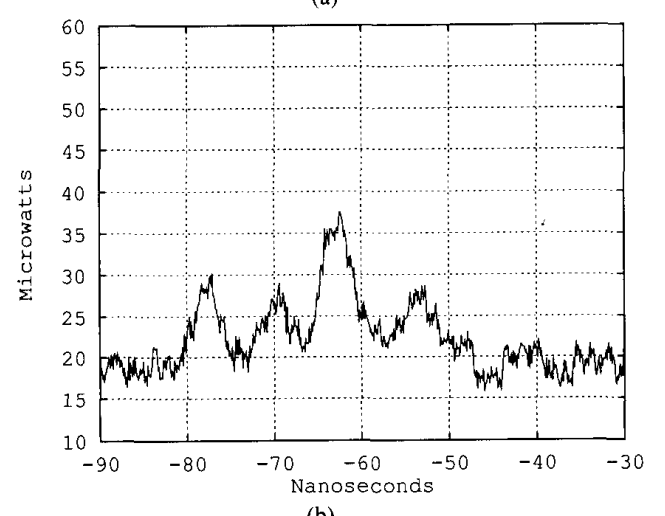

(b)

Fig. 8. (a) Selection of D1, D2, D3, D4 measured at detector D1, (b) selection of D1, D2, D3, D4 measured at detector D3.

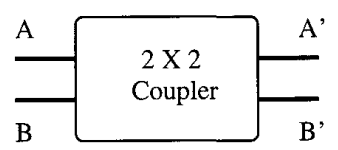

Fig. 9. Symmetric fiber coupler.

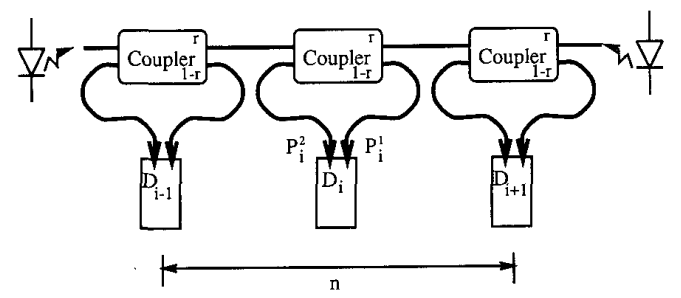

Fig. 10. Linear optical bus.

Since the bus is symmetrical, we can analyze one signal that originates on the left from a single transmitter and propagates to the right as shown in Fig. 10.

Fig. 11 is a plot of $p_{i}^{1}$ versus $i$ for various values of $r$. Note that the values of $i$ are plotted on a logarithmic scale. The topmost curve is for a bus with $r=90 \%$ where the power at the first detector is $10 \%$ of the initial power. The lowest curve is for a bus with $r=99 \%$ where power at the first detector is $1 \%$ of the initial pulse power. For all

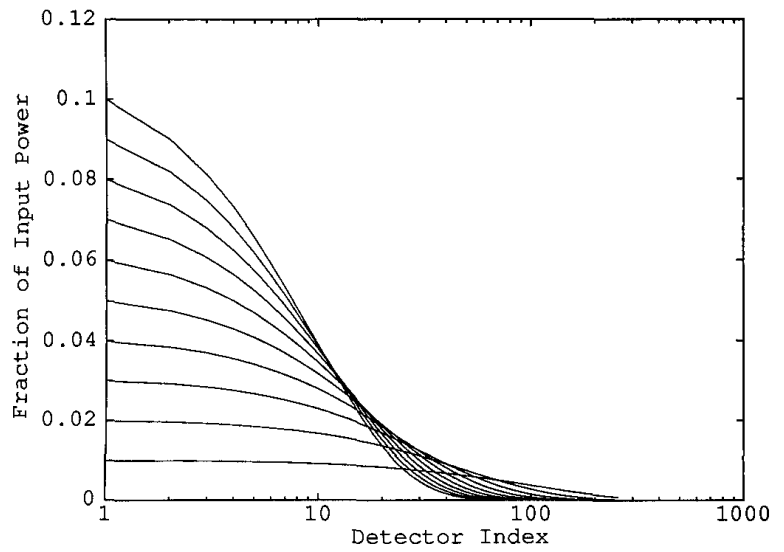

Fig. 11. Power $p_{i}^{\prime}$ at detector $D_{i}$ for $90 \% \leq r \leq 99 \%$.

the curves, the absolute power falls off geometrically with increasing $i, 1 \leq i \leq n$.

A bound on the number of detectors, $n$ is determined by the sensitivity of the last detector on the bus. In other words, it is the bound for a detector to discriminate between "no pulse" and "pulse." If the last detector has a sensitivity $P \min$, then the maximum number of detectors supportable is

$$
n=\frac{\log \left(\frac{P \min }{1-r}\right)}{\log (r)}+1 .
$$

Equation (4) is shown graphically in Fig. 12 for a set of coupling ratios $r=90 \%, 95 \%, 97 \%, 98 \%, 99 \%$, and $0.01 \% \leq P \min \leq 1 \%$ of the input power on a logarithmic scale. This graph confirms the intuition that by improving either the coupling ratio $r$, or the sensitivity of the detectors $P \min$, we will be able to support more detectors on the bus. We also note the sharp drop in $n$ for high values of $P \min$ and $r$, which reflects the situation where much of the available power flows off the end of the bus and is wasted.

However, for our experimental setup, it is clear that it is not the absolute power but rather the power margin that imposes a bound on the size of the system. In addition, since the bus configuration chosen for this structure requires bidirectional propagation, we are constrained to use a single tapping ratio, $r$, for all couplers. Based on these two constants, the graph shown in Fig. 13, which is a plot of worst-case power margin $P m$ versus $1-r$ for various bus lengths, confirms that the power margin for the coincident structure bounds scalability more strongly than absolute power. We can see from Fig. 12 that using commercially available $95 \%$ couplers, and assuming we can tolerate a $P$ min of 0.0001 of input power we could achieve bus lengths of abut 120 detectors. This would be the case for an input of $100 \mathrm{~mW}$ of power injected into the bus, and a detector sensitivity of $10 \mu \mathrm{W}$, operating at 250 $\mathrm{MHz}$. However, Fig. 13 shows that for a power margin of $P m=20 \%$ we could only reach lengths of 32 detec- 


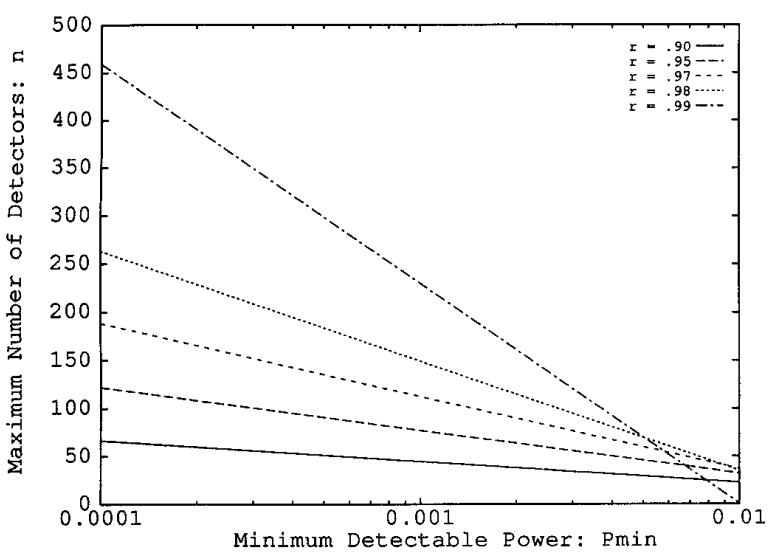

Fig. 12. Number of detectors versus $P \min$ for various values of $r$.

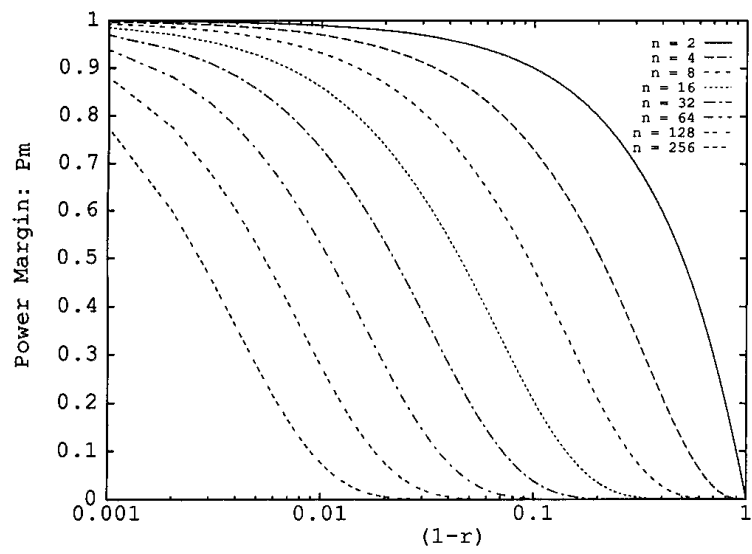

Fig. 13. Power margin $P_{m}$ versus $0.001 \geq(1-r) \geq 1$ for various bus sizes.

tors. Therefore, due to both minimum power constraints and power margin issues the system scale is highly sensitive to the fixed value of $r$. Further, we note that power margin imposes a tighter constraint than absolute power.

To help alleviate this problem, we propose a two level bus structure. By using two levels, we can essentially increase the tapping ratios on our buses and more effectively control the amount of power at each detector.

\section{B. The Dual-Level Structure}

The basis for the power distribution problem in the linear system is the fact that detectors at the start of the bus use more power than needed and, therefore, detectors at the end of the bus are starved. If we were to relax the requirement of fixed ratio taps in favor of varying the coupling ratios, we would need a number of distinct, precisely tuned couplers approaching the number of detector sites [9]. Yet, no couplers exist that would allow tuning to a precision of more than one or two percent. Of course, the use of tuned couplers forces the network to be unidirectional since coupling ratios must decrease in the direction of propagation.

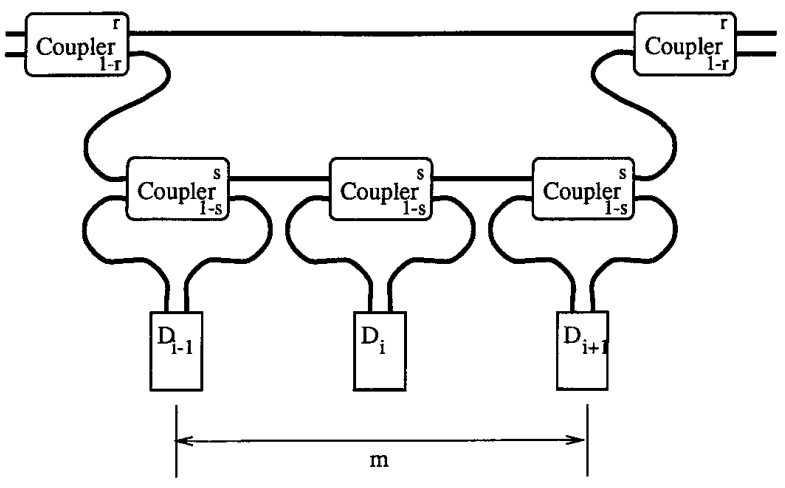

Fig. 14. Dual-level optical bus.

An alternative method that does not require multiple coupling ratios is to adopt a dual-level bus structure. As shown in Fig. 14, we split the bus into a main fiber and a sublevel to create a section of the bus, labeled $m$. The sublevel contains $m$ detectors in a linear arrangement except for the last detector, which feeds back the remaining power into the main fiber and the next section. In the main fiber, care must be taken to ensure that the optical path length is the same as the subsection so that the two parts of the signal arrive synchronized at the next section. The dual-level bus consists of a series of these sections.

Once again, we start with an analysis of absolute power for this structure, and then proceed to power margin issues. Thus, we assume the input is from the left (into the upper leg to the first coupler) and propagates to the right. The detectors are numbered linearly in the direction of propagation.

We further assume two types of couplers with splitting ratios of $r$ and $s$ for the main level and sublevel, respectively. The power at any given detector site in Fig. 14 is given by

$$
p_{i}=\left(\begin{array}{ll}
1-r & r
\end{array}\right) A^{k}\left(\begin{array}{l}
1 \\
0
\end{array}\right) s^{l}(1-s)
$$

where $p_{i}$ is the power at site $i, r$, and $s$ are coupling ratios, $k$ is $i$ div $m, l$ is $i \bmod m, m$ is the number of detectors in a sublevel, and

$$
A=\left(\begin{array}{ll}
r & 1-r \\
(1-r) s^{m} & r s^{m}
\end{array}\right) .
$$

From linear algebra [11], we know that a vector of the form $u_{k}=A^{k} u_{o}$ can be rewritten as $u_{k}=\sum_{i=1}^{n} c_{i} \lambda_{i}^{k} x_{i}$, where $\lambda_{i}$ are the eigenvalues of matrix $A$, the $x_{i}$ 's are the associated eigenvectors and the coefficients $c_{i}$ are determined from the initial condition $u_{o}$.

For our analysis, we rewrite the matrix of (5) in the form

$$
A^{k}\left(\begin{array}{l}
1 \\
0
\end{array}\right)=c_{i} \lambda_{1}^{k} x_{1}+c_{2} \lambda_{2}^{k} x_{2}
$$

and the coefficients are determined by $c_{1} x_{1}+c_{2} x_{2}=u_{o}$. 
The $x_{i}$ are vectors and they are given by $x_{i}=\left(\begin{array}{l}\xi_{i} \\ i\end{array}\right)$. Rewriting the coefficient equation gives

$$
\left(\begin{array}{c}
c_{1} \xi_{1} \\
c 1
\end{array}\right)+\left(\begin{array}{c}
c_{2} \xi_{2} \\
c 2
\end{array}\right)=\left(\begin{array}{l}
1 \\
0
\end{array}\right)
$$

which has the solution

$$
c_{1}=\frac{1}{\xi_{1}-\xi_{2}} .
$$

Assuming, without loss of generality, that $\lambda_{1}>\lambda_{2}$ as $k$ increases, then the $c_{1} \lambda_{1}^{k} x_{1}$ term in (7) quickly dominates. Therefore, a good approximation is given by

$$
p_{i}=\left[\begin{array}{ll}
(1-r) & r
\end{array}\right] c_{1} \lambda_{1}^{k} x_{1} s^{l}(1-s) .
$$

Fig. 15 shows a comparison of a linear bus and a duallevel structure for the particular case of $r=s=90 \%, n$ $=256$, and $m=\operatorname{sqrt}(n)$. Clearly, the power at the detectors for the linear bus falls off much more rapidly than for the dual-level bus. The dual-level bus shows a characteristic "saw-tooth" pattern of power distribution. At the beginning of each section, power is restored by injection of power from the main backbone. This more evenly distributes all of the available power down the length of the bus.

In the linear structure, we examined the bounds for the minimum power needed at the last detector. For the duallevel structure, we will examine the minimum power seen at the last detector of the last section. This minimum power is given by the equation

$$
P \min =\lambda_{1}^{k}\left(\xi_{1}(1-r)+r\right) c_{1} s^{m-1}(1-s) .
$$

As with the linear case, the ability to support large systems is dependent upon maximizing the values of $r$ and $s$. However, in the dual-level case, we additionally may vary $m$, the number of detectors per section. The relationship between $r, s$, and $m$ is captured in $\lambda_{1}$, which is a monotonically increasing function of $r$, and $s$ but is not monotonic in $m$. Therefore, it is desirable to fix $r$ and $s$ to be as large as possible and adjust $m$ to maximize the total number of detectors in the system.

This relationship is shown in Fig. 16. The two families of curves represent coupling ratios of $r=s=90 \%$ and $r=s=95 \%$. The curves are the number of detectors (length of the bus) supportable at different $P$ min values. For the $90 \%$ curves, $P \min =0.0001,0.0002,0.0004$, $0.0008,0.0016,0.0032$, and 0.0064 . For the $95 \%$ curves, $P_{\min }=0.0001,0.0002,0.0004,0.0008$, and 0.0016 . Note that the dual-level structure with $95 \%$ couplers cannot support high minimum power detectors since the power into the first detector $p_{1}=0.05 \times 0.05=0.0025$. The long tails on the curves reflect the condition where $m$ $\geq n$.

Having chosen values for $r, s$, and $m$, we can rewrite equation (9) to compute the number of detectors supportable as a function of $P$ min:

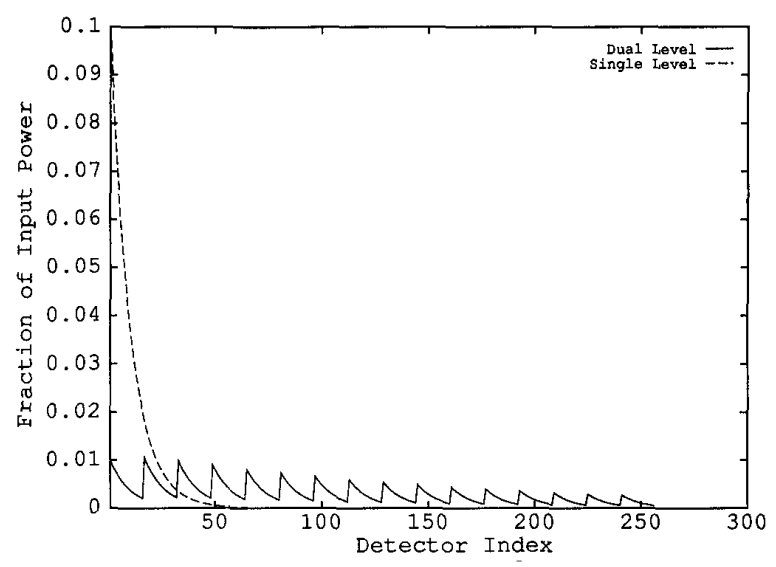

Fig. 15. Power at detector sites for single- and dual-level 256 node buses.

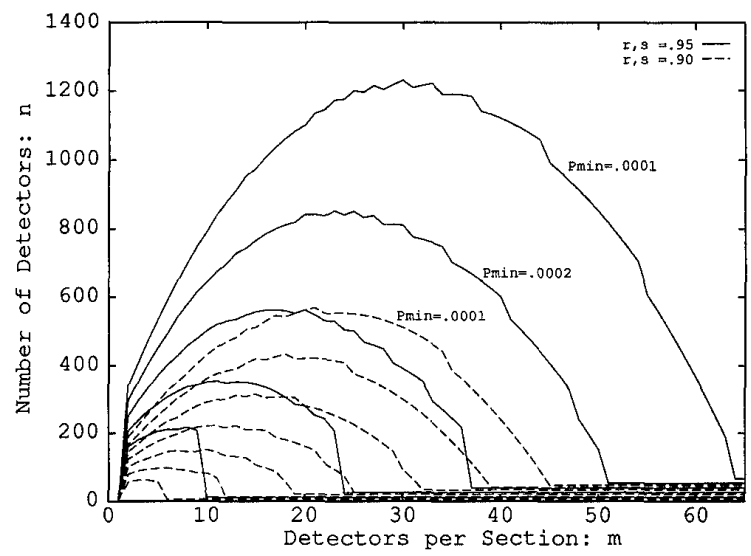

Fig. 16. $n$ versus $m$ for $r, s=0.90,0.95$, and various $P \min$.

$$
n=m \frac{\log \left(\frac{P \min }{\left((1-r) x_{1}+r\right) c_{1} s^{k-1}(1-s)}\right)}{\log \left(\lambda_{1}\right)} .
$$

A plot of numerical solutions for (10) is shown in Fig. 17.

Equation (10) and Fig. 17 allow a direct comparison of the dual-level bus performance shown in Fig. 17 with linear bus performance derived in (4) and plotted in Fig. 12. From this comparison, we can see that, in terms of $P$ min, the optimized dual level bus gives approximate factors of between 4 and 10 improvement (depending on the coupler ratios) over the simple linear configuration.

To perform the analysis of power margin for the two level structure, we compare the maximum power at any detector to the minimum power at any detector on the bus. This simplifies the calculation and gives a bound on the "envelope" of the saw-tooth power curve (as shown in Fig. 15). For these curves (shown in Fig. 18) we are again using $m=s q r t(n)$ and $r=s$. Unlike the curves for the linear bus, these curves have a peak and approach an asymptotic value for very large values of $r$ and $s$. This is 


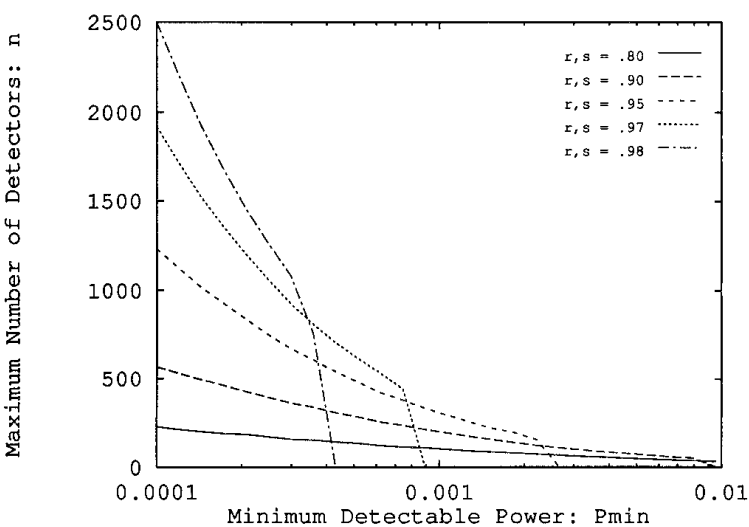

Fig. 17. Pmin versus $n$ for different values of $r, s$.

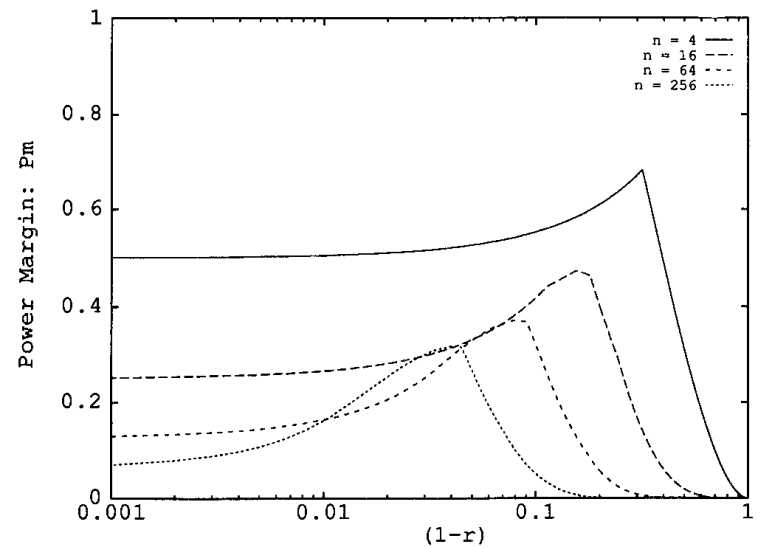

Fig. 18. $P m$ versus $0.001 \geq(1-r)=(1-s) \geq 0$ for dual-level buses.

because, similar to the linear case, we reach a point at which a significant percentage of the power must be thrown away at the end of the bus, in order to account for the large coupling ratio of the final tap. However, it is still the case that $P m$, the power maximum margin, limits the scalability of the system more tightly than absolute power. As a practical example similar to the linear case, using available $95 \%$ percent couplers, the power margin limits bus size to about 300 detectors, rather than the 1250 detectors we could expect based on minimum power requirements of $P_{\min }=0.0001$

\section{Summary}

Clearly, three factors, threshold power margin, synchronization error, and coupling ratio determine system scale. Our experiments have shown that the important system issues of latency and throughput which are related to pulse width limits are highly scalable. Based on current and near term technology, we have shown that synchronization error does not contribute significantly to the bounds calculated above.

On the other hand, physical scalability issues such as the size of the bus and the number of detectors that can be supported are more severely restricted due to power distribution in a system built from passive couplers. However, we believe near term technologies (e.g., fiber amplifiers) and alternate bus structures will alleviate this problem. The fact that the temporal scalability bounds show significantly shorter pulses can be supported, is very encouraging for the long-term application of this technique.

\section{REFERENCES}

[1] F. C. Allard, Fiber Optics Handbook For Engineers and Scientists. New York: McGraw-Hill, 1990.

[2] D. M. Chiarulli, S. P. Levitan, and R. G. Melhem, "Self routing interconnection structures using coincident pulse techniques, " in SPIE OE/Boston'90 (Boston, MA), Nov. 4-9, 1990.

[3] D. M. Chiarulli, R. G. Melhem, and S. P. Levitan, "Parallel memory using coincident optical pulses," IEEE Comput., vol. 20, no. 12, pp. 48-57, Dec. 1987.

[4] C. R. Giles, E. Desurvire, J. R. Talman, J.R. Simpson, and P. C Becker, "2-Gb/s signal amplification at $\lambda=1.53 \mu \mathrm{m}$ in a erbiumdoped single-mode fiber amplifier," J. Lightwave Technol., vol. 7 no. 4, pp. 651-656, Apr. 1989.

[5] Gould Electronics, Glenn Burnie, MD, Gould Fiber Optics Technical Notes.

[6] R. I. Laming et al., "Efficient pump wavelengths of erbium-doped fiber optical amplifiers," Electron. Lett., vol. 25, no. 1, pp. 12-14, Jan. 1989.

[7] S. P. Levitan, D. M. Chiarulli, and R. C. Melhem, "Coincident putse techniques for multiprocessor interconnection structures," Appl. Opt., vol. 29, no. 14, pp. 2024-2033, May 1990.

[8] R. G. Melhem, D. M. Chiarulli, and S. P. Levitan, "Space multiplexing of optical waveguides in a distributed multiprocessor, " Comput. J. British Comput. Society, vol. 32, no. 4, pp. 362-369, 1989.

[9] M. Nassehi, F. Tobagi, and M. Marhic, "Fiber optic configurations for local area networks," IEEE J. Select. Areas Commun., vol. SAC-3, no. 6, pp. 941-949, Nov. 1985.

[10] D. Sarrazin, H. Jordan, and V. Heuring, "Digital fiber optic delay line memory," in Digital Optical Computing II, vol. 1215 (Los Angeles, CA), Jan. 1990. SPIE.

[11] Gilbert Strang, Linear Algebra and Its Applications, 2nd ed. New York: Academic, 1980.

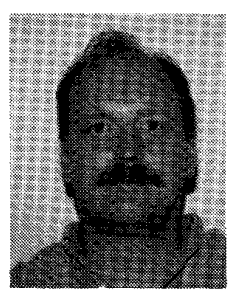

Donald M. Chiarulli received the B.S. degree in physics from Louisiana State University in 1976. the M.S. degree in computer science from Virginia Polytechnic Institute, Blacksburg, in 1979. and the Ph.D. degree in computer science from Louisiana State University in 1986.

$\mathrm{He}$ is an Assistant Professor of Computer Science at the University of Pittsburgh. His research interests include architectures and algorithms for the design and implementation of highly parallel computing systems, and optical technologies for interconnection networks and processing.

Dr. Chiarulli is a member of the IEEE Computer Society, SPIE, and the Optical Society of America.

Robert M. Ditmore, photograph and biography not available at the time of publication. 


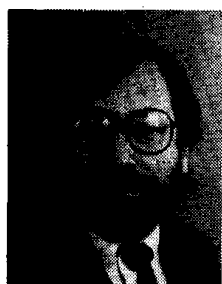

Steven P. Levitan (S'83-M'83) received the B.S. degree from Case Western Reserve University in 1972 and the M.S. and Ph.D. degrees both in computer science, from the University of Massachusetts, Amherst in 1979 and 1984, respectively.

He is the Wellington C. Carl Assistant Professor of Electrical Engineering at the University of Pittsburgh, Pittsburgh, PA. He was an Assistant Professor from 1984 to 1986 in the Electrical and Computer Engineering Department at the University of Massachusetts. In 1987 he joined the Electrical Engineering faculty at the University of Pittsburgh. His research interests include optical computing, parallel computer architecture, paralle algorithm design, and computer aided design for VLSI.

Dr. Levitan is a member of the IEEE Computer Society, ACM, SPIE, and OSA.

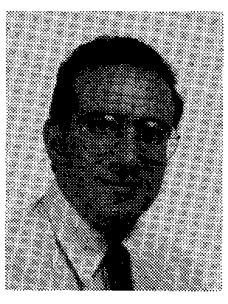

Rami G. Melhem received the B.E. degree in electrical engineering from Cairo University, Egypt, in 1976, the M.S. degree in mathematics/ computer science from the University of Pittsburgh in 1981 , and the $\mathrm{Ph} . \mathrm{D}$. in computer science from the University of Pittsburgh in December 1983.

$\mathrm{He}$ is an Associate Professor of Computer Science at the University of Pittsburgh, Pittsburgh, PA. He has been an Assistant Professor of Computer Science at Purdue University from 1984 to 1986 and at the University of Pittsburgh from 1986 to 1989 . His research interests include optical computing parallel systems, fault tolerant systems and the application of large computational arrays to scientific problems. 